\title{
A Fast, Simple, and Affordable Technique to Measure Oxygen Consumption in Living Zebrafish Embryos
}

\author{
Julie Somkhit, ${ }^{1, *}$ Roxane Loyant, ${ }^{2, *}$ Alexandre Brenet, ${ }^{1}$ Rahma Hassan-Abdi, \\ Constantin Yanicostas, ${ }^{1}$ Mathieu Porceddu, \\ Annie Borgne-Sanchez, ${ }^{2}$ and Nadia Soussi-Yanicostas ${ }^{1}$
}

\begin{abstract}
In all animal species, oxygen consumption is a key process that is partially impaired in a large number of pathological situations and thus provides informative details on the physiopathology of the disease. In this study, we describe a simple and affordable method to precisely measure oxygen consumption in living zebrafish larvae using a spectrofluorometer and the MitoXpress Xtra Oxygen Consumption Assay. In addition, we used zebrafish larvae treated with mitochondrial respiratory chain inhibitors, antimycin A or rotenone, to verify that our method enables precise and reliable measurements of oxygen consumption.
\end{abstract}

Keywords: respiration, zebrafish larvae, oxygen consumption

\section{Introduction}

$\mathbf{R}$ ESPIRATION Is A KEY cellular function, which is altered in a great number of disorders and animal disease models, thus providing a meaningful window to interrogate the physiology of living cells and organisms. ${ }^{1}$ Automated devices allowing precise measurement of both mitochondrial respiration (oxidative phosphorylation) and glycolysis have been developed in recent years, but their price and cost of usage restrain their use on a daily basis. To overcome this limitation, we describe here a simple and affordable method that allows precise measurement of oxygen consumption in living zebrafish larvae, using a simple spectrofluorometer and the MitoXpress Xtra Oxygen Consumption Assay (Agilent), a quantitative test relying on a fluorescent probe, which is quenched in a dose-dependent manner by $\mathrm{O}_{2}$ molecules (Fig. 1A).

\section{Results}

To assess the ability of this method to detect fine changes in $\mathrm{O}_{2}$ consumption in living zebrafish larvae, we measured $\mathrm{O}_{2}$ consumption of pools of seven 5 days post-fertilization wild-type individuals treated with either vehicle (1\% dimethyl

\footnotetext{
${ }^{1}$ NeuroDiderot, Inserm U1141, Université de Paris, Paris, France.

${ }^{2}$ Mitologics SAS, Faculté de Médecine, Créteil, France.

*These authors contributed equally to this work.
}

(C) Julie Somkhit et al. 2020; Published by Mary Ann Liebert, Inc. This Open Access article is distributed under the terms of the Creative Commons Attribution Noncommercial License (http://creativecommons.org/licenses/by-nc/4.0/) which permits any noncommercial use, distribution, and reproduction in any medium, provided the original author(s) and the source are cited. 


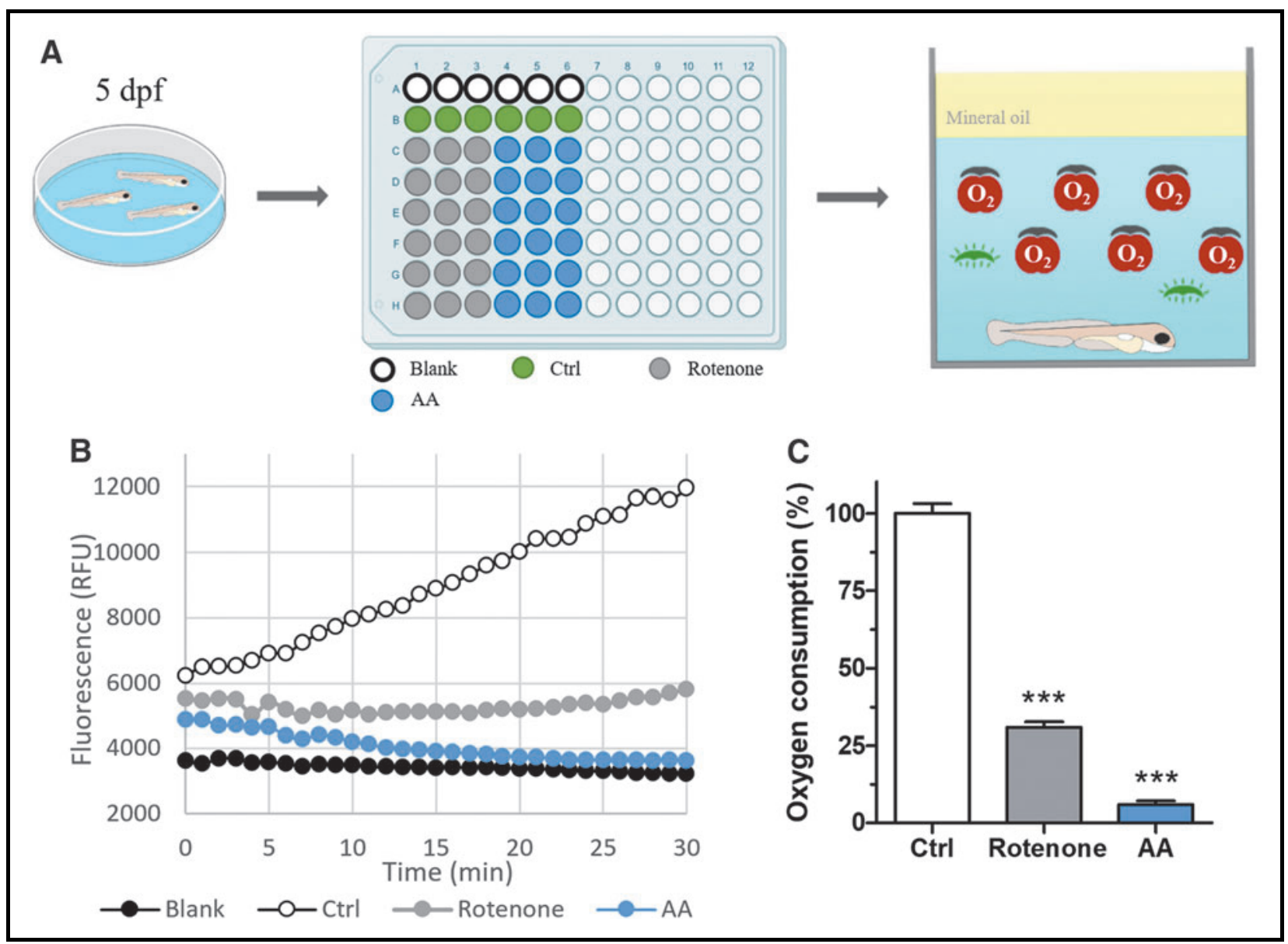

FIG. 1. Real-time measurement of oxygen consumption in 5 dpf zebrafish larvae. (A) Scheme of the experimental setup used to measure oxygen consumption in vivo in zebrafish larvae, treated or not with MRC inhibitors, with an example of a filled 96-well microplate. Pools of seven 5 dpf larvae treated with MRC inhibitors or untreated (control) were transferred to a 96-well microplate (Greiner Bio-One International) and then incubated in the presence of the MitoXpress Xtra probe, (Supplementary Data) a fluorescent molecule whose fluorescence is quenched in a dose-dependent manner by $\mathrm{O}_{2}$ through direct molecular interactions, thus making fluorescence intensity inversely proportional to the amount of free $\mathrm{O}_{2}$ dissolved in the medium. (B) Real-time measurement of the oxygen consumption in larvae exposed to MRC inhibitors or untreated, and blank (E3 medium without larva). (C) Quantification of the oxygen consumption of larvae exposed to either $2 \mu \mathrm{M}$ rotenone or $10 \mu \mathrm{M}$ AA. Whiskers represent the standard error of the mean. Rotenone $(n=36)$, AA $(n=35)$, control $(n=18)$, and blank $(n=24)$, one-way ANOVA with Tukey's Multiple Comparison Test: $* * * p<0.0001$. AA, antimycin A; ANOVA, analysis of variance; dpf, days post-fertilization; MRC, mitochondrial respiratory chain.

sulfoxide [DMSO]), $2 \mu \mathrm{M}$ rotenone, a specific inhibitor of the mitochondrial respiratory chain (MRC) complex $\mathrm{I}^{2},{ }^{2}$ or $10 \mu \mathrm{M}$ antimycin A (AA), a specific inhibitor of the MRC complex III (Fig. 1A). ${ }^{3,4}$ As previously reported, we found that $1 \%$ DMSO concentration had no effect on larval development. ${ }^{5}$

Results showed that oxygen consumption of larvae exposed to $2 \mu \mathrm{M}$ rotenone and $10 \mu \mathrm{M}$ AA was $31 \%$ and $6 \%$, respectively, of that observed in non-treated controls (Fig. 1B, C, Supplementary Data), indicating that the method described allows reliable measurements of oxygen consumption. Thus, this simple and direct method can be used to measure respiration in vivo in zebrafish intoxication and disease models.

\section{Disclosure Statement}

No competing financial interests exist. 


\section{Funding Information}

This study was supported by a grant from the Agence Nationale de la Recherche (ANR-16-CE18-0010 MITOXDRUGS).

\section{Supplementary Material}

Supplementary Data

\section{References}

1. Frazier AE, Thorburn DR, Compton AG. Mitochondrial energy generation disorders: genes, mechanisms, and clues to pathology. J Biol Chem 2019;294:5386-5395.

2. Lindahl PE, Öberg KE. The effect of rotenone on respiration and its point of attack. Exp Cell Res 1961;23:228-237.

3. Huang LS, Cobessi D, Tung EY, Berry EA. Binding of the respiratory chain inhibitor antimycin to the mitochondrial Bc1 complex: a new crystal structure reveals an altered intramolecular hydrogen-bonding pattern. J Mol Biol 2005;351:573-597.

4. Rieske JS. Inhibitors of respiration at energy-coupling site 2 of the respiratory chain. Pharmacol Ther 1980;11:415-450.

5. Alavi Naini SM, Yanicostas C, Hassan-Abdi R, Blondeel S, Bennis M, Weiss RJ, et al. Surfen and oxalyl surfen decrease tau hyperphosphorylation and mitigate neuron deficits in vivo in a vertebrate model of tauopathy. Transl Neurodegener 2018;7:6.

Address correspondence to:

Nadia Soussi-Yanicostas NeuroDiderot, INSERM U1141

Hôpital Robert Debré

48 Boulevard Sérurier

Paris F-75019

France

E-mail: nadia.soussi@inserm.fr 